Vol. 44 (1991) [71-78]

\title{
ON THE $\ell$-ADIC REPRESENTATIONS ATTACHED TO SIMPLE ABELIAN VARIETIES OF TYPE IV
}

\author{
Wenchen ChI
}

The $\ell$-adic representations associated to prime dimensional type IV absolutely simple abelian varieties over number fields are studied. The image of such a representation was computed. The results coincide with the well-known conjectures of Mumford and Tate.

\section{INTRODUCTION}

Let $K$ be an algebraic number field and let $\bar{K}$ be an algebraic closure of $K$. Let $G_{K}=\operatorname{Gal}(\bar{K} / K)$. For an abelian variety $A$ defined over $K$, we denote by $\operatorname{End}^{\circ}(A)$ the endomorphism algebra $\operatorname{End}_{\bar{K}}(A) \otimes_{\mathrm{Z}} \mathbb{Q}$ of $A$. For each prime number $\ell$, let $T_{\ell}$ be the Tate module of $A$ and let $V_{\ell}=T_{\ell} \otimes z_{\ell} \mathbb{Q}_{\ell}$. The Galois group $G_{K}$ acts continuously on $T_{\ell}$. One has the $\ell$-adic representation $\rho_{\ell}: G_{K} \rightarrow \operatorname{Aut}\left(V_{\ell}\right)$.

According to Albert's classification of division algebras with positive involutions, the so-called type IV absolutely simple abelian varieties over $K$ are those abelian varieties $A$ with $D=\operatorname{End}^{\circ}(A)$ is a division algebra over its centre $E$, where $E$ is a $C M$-field. Let $\dot{E}^{+}$be the maximal totally real subfield of $E$. If $[D: E]=f^{2}$ and $\left[E^{+}: \mathbb{Q}\right]=e$, then $e f^{2}$ divides $\operatorname{dim} A$ (see [6], Section 21). In particular, when $\operatorname{dim} A=p$ is a prime number, it is easy to see that $D=E$ and $E$ is a $C M$-field of degree $2 p$ or an imaginary quadratic field. Existence of such type of abelian varieties over number fields except the case where $\operatorname{dim} A=2$ and $E$ is an imaginary quadratic field was proved by Shimura in [12].

In this paper, we are interested in the $\ell$-adic representations associated to the above prime dimensional type IV absolutely simple abelian varieties over number fields. For the case where $E$ is a $C M$-field of $2 \operatorname{dim} A$, the $\mathbb{Q}_{\ell}$-Lie algebra $\mathcal{G}_{\ell}$ of the image of the $\ell$-adic representation is well-known to be equal to $\mathcal{M}_{\ell}=\mathcal{M} \otimes \mathbb{Q} \mathbb{Q}_{\ell}$, where $\mathcal{M}$ is the Q-Lie algebra of the Mumford-Tate group associated to $A$ (thought of as over $\mathbb{C}$ ). This is due to Taniyama and Shimura in [11]. In the sequel, we shall study the remaining cases. Namely, $\operatorname{dim} A=p$ is an odd prime number and $\operatorname{End}^{\circ}(A)=E$ is an imaginary quadratic field.

Received 7 August 1990

Supported by N.S.C., R.O.C.

Copyright Clearance Centre, Inc. Serial-fee code: 0004-9729/91 \$A2.00+0.00. 
Fix a $K$-polarisation on $A$ once for all. Let $\psi$ be the associated Riemann form on $V_{\ell}$. The induced Rosati involution on $E$ is the complex conjugation. One has $\psi(\alpha v, w)=\psi(v, \bar{\alpha} w)$ for $\alpha$ in $E$ and $v, w$ in $V_{\ell}$. The Tate module $V_{l}$ is a free $E_{\ell}=E \otimes \mathbb{Q} \mathbb{Q}_{\ell}$-module of rank $p$. Let $G_{V_{\ell}}$ be the algebraic envelope of the $\ell$-adic Lie group $G_{\ell}=\operatorname{Im} \rho_{\ell}$. By the theorem of Faltings ([4], Section 5, Satz 3), $G_{V_{\ell}}$ is a reductive algebraic group over $\mathbb{Q}_{l}$. Let $S_{V_{l}}$ be the connected component of the identity of $G_{V_{l}} \cap S L_{V_{\ell}}$ and let $\mathcal{S}_{\ell}$ be its Lie algebra. By replacing the base field $K$ by a finite extension, we may assume that $\operatorname{End}_{\bar{K}}(A)=\operatorname{End}_{K}(A)$. Then $G_{V_{\ell}}\left(\mathbb{Q}_{\ell}\right)$ is contained in the commutant of $E_{\ell}$ in the symplectic similitudes $G S p\left(V_{\ell}, \psi\right)$. On the other hand, let $\alpha$ be a nonzero element in $E$ such that $\bar{\alpha}=-\alpha$. It can be shown that there is a unique $E_{\ell}$-Hermitian form $\phi$ on $V_{\ell}$ such that

$$
\psi(v, w)=\operatorname{Tr}_{E_{\ell / Q_{\ell}}}(\alpha \phi(v, w)) \text { for all } v, w \text { in } V_{\ell} .
$$

The commutant of $E_{\ell}$ in the symplectic group $S p\left(V_{\ell}, \psi\right)$ is easily seen to be the unitary group $U\left(V_{\ell / E_{\ell}}, \phi\right)$, which can be regarded as an algebraic group over $\mathbb{Q}_{\ell}$.

By an $\ell$-adic analogy to the method in $[7,13]$, we shall prove that, for all prime dimensional absolutely simple abelian varieties of type IV over number fields, the reductive Lie algebra $\mathcal{S}_{\ell}$ is equal to the Lie algebra of $U\left(V_{\ell / E_{\ell}}, \phi\right)$. In particular, $\mathcal{G}_{\ell}=\mathcal{M} \otimes_{\mathbb{Q}} \mathbb{Q}_{\ell}$ as was conjectured in [5]. Consequently, the conjecture of Tate on algebraic cycles (see [15]) is true for all prime dimensional absolutely simple abelian varieties of type IV over number fields.

\section{Preliminaries}

\subsection{The algebraic envelope $G_{V_{l}}$.}

Let $A$ be an abelian variety defined over a number field $K$. For each prime number $\ell$, let $\rho_{\ell}: G_{K} \rightarrow \operatorname{Aut}\left(V_{\ell}\right)$ be the associated $\ell$-adic representation. The image $G_{\ell}$ of $\rho_{\ell}$ is then an $\ell$-adic Lie group. Let $\mathcal{G}_{\ell}$ be the Lie algebra of $G_{\ell}$. It is easily seen that $\mathcal{G}_{\ell}$ is invariant under finite extensions of the base field $K$.

Let $G_{V_{\ell}}$ be the algebraic envelope of $G_{\ell}$, that is, $G_{V_{\ell}}$ is the smallest algebraic subgroup of $G L_{V_{l}}$ defined over $\mathbb{Q}_{\ell}$ such that $G_{l}$ is contained in $G_{V_{l}}\left(\mathbb{Q}_{\ell}\right)$. By the Theorems of Faltings ([4], Section 5, Satz 3,4), $\mathcal{G}_{\ell}$ (respectively $G_{V_{l}}$ ) is reductive and $\operatorname{End}_{\mathcal{O}_{\ell}}\left(V_{\ell}\right)=\operatorname{End}_{K}(A) \otimes \mathbf{z} \mathbb{Q}_{\ell}\left(\right.$ respectively $\operatorname{End}_{G_{V_{\ell}}\left(Q_{\ell}\right)}\left(V_{\ell}\right)=\operatorname{End}_{K}(A) \otimes_{\mathbf{Z}} \mathbb{Q}_{\ell}$ ). On the other hand, Bogomolov ([1], Corollary 1) proved that $\mathcal{G}_{\ell}$ (respectively $G_{V_{\ell}}$ ) contains the homotheties $\mathbb{Q}_{\ell}$ (respectively $G_{m}$ ). Replacing the base field $K$ by a finite extension of $K$, we may assume that $G_{V_{l}}$ is connected (see [2], Section 3.3). Let $S_{V_{l}}$ be the connected component of the identity of $G_{V_{l}} \cap S L_{V_{l}}$. Then $S_{V_{l}}$ is again a connected reductive algebraic subgroup of $G L_{V_{l}}$ defined over $Q_{\ell}$. Then $G_{V_{l}}=S_{V_{l}} \cdot G_{m}$. Let $S_{\ell}$ be the Lie algebra of $S_{V_{\ell}}$. Then $\mathcal{S}_{\ell}$ is a reductive Lie algebra over $\mathbb{Q}_{\ell}$ and $\mathcal{G}_{\ell}=\mathcal{S}_{\ell} \oplus \mathbb{Q}_{\ell}$. 


\subsection{The Hodge-Tate decomposition of $V_{l}$. (see $[8,10]$ )}

Let $C_{\ell}$ be the completion of a fixed algebraic closure of $\mathbb{Q}_{\ell}$ and let $S_{\ell}$ be the set of all finite places of $K$ dividing $\ell$. For each $v \in S_{\ell}$, let $\bar{K}_{v}$ be the algebraic closure of $K_{v}$ in $C_{\ell}$. As a $\mathrm{Gal}\left(\bar{K}_{v} / K_{v}\right)$-module, it is well-known that $V_{\ell}$ is a Hodge-Tate module of weights 0 and 1 , each of them with multiplicity $\operatorname{dim} A$ (due to Tate and Raynaud, see [10], p.157). Denote the Hodge-Tate decomposition of $V_{\ell}$ by $V_{\ell} \otimes_{Q_{\ell}} C_{\ell}=$ $V_{C_{\ell}}(0) \oplus V_{C_{\ell}}(1)$. More precisely, $V_{C_{\ell}}(0)$ is the cotangent space (over $C_{\ell}$ ) to the dual abelian variety $A$ of $A$ at its origin and $V_{C_{l}}(1)$ is the 1-fold Tate twist of the tangent space (over $C_{\ell}$ ) to $A$ at its origin (see [16], Corollary 2 of Theorem 3).

For each $v \in S_{\ell}$, let $\bar{v}$ be an extension of $v$ to $\bar{K}$. Then the local Galois group $\operatorname{Gal}\left(\bar{K}_{v} / K_{v}\right)$ can be identified with the decomposition group $D_{\bar{v}}$ for $\bar{v}$ in $\mathrm{Gal}(\bar{K} / K)$. Let $I_{\bar{v}}$ be the inertia subgroup of $D_{\bar{v}}$. Then the algebraic envelope of $\rho_{\ell}\left(I_{\bar{v}}\right)$ is an algebraic subgroup of $G_{V_{\ell}}$. By a theorem of Sen ([8], Section 6), the one-parameter subgroup $h_{V_{\ell}}$ of $G L_{V_{\ell / C_{\ell}}}$ defined by

$$
h_{V_{l}}(c)(x)= \begin{cases}x, & \text { if } x \in V_{C_{\ell}}(0) \\ c x, & \text { if } x \in V_{C_{\ell}}(1)\end{cases}
$$

maps $G_{m / C_{\ell}}$ into the algebraic envelope of $\rho_{\ell}\left(I_{\bar{v}}\right)$ over $C_{\ell}$. So $h_{V_{\ell}}$ is a one-parameter subgroup of $G_{V_{\ell}}$ defined over $C_{\ell}$.

\subsection{The UNITARY GROUP $U\left(V_{\ell / E_{\ell}}, \phi\right)$.}

For our purpose, we now assume that $E=\operatorname{End}^{\circ}(A)$ is an imaginary quadratic field. For a fixed $K$-polarisation on $A$, let $\psi$ be the associated Riemann form on $V_{l}$. The induced Rosati involution on $E$ is the complex conjugation. Let $E_{\ell}=E \otimes_{\mathbb{Q}} \mathbb{Q}_{\ell}$.

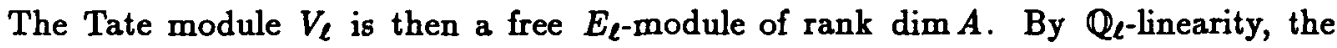
complex conjugation on $E$ extends to an involution on the $\mathbb{Q}_{\ell}$-algebra $E_{\ell}$. We denote it again by - . Then

$$
\psi(\alpha v, w)=\psi(v, \bar{\alpha} w) \text { for all } v, w \text { in } V_{\ell} ; \alpha \text { in } E_{\ell} .
$$

Let $\operatorname{Tr}_{E_{\ell / Q_{\ell}}}$ be the regular trace of $E_{\ell}$ over $\mathbb{Q}_{\ell}$. The following results are an analogy of Lemmas 4.6, 4.7 in [3].

LemMa 2.1. Let $V$ and $W$ be free $E_{\ell}$-modules of finite rank and let $\psi: V \times$ $W \rightarrow \mathbb{Q}_{\ell}$ be a $\mathbb{Q}_{\ell}$-bilinear form such that $\psi(e v, w)=\psi(v, e w)$ for all $e$ in $E_{\ell}, v$ in $V$, and $w$ in $W$. Then there exists a unique $E_{\ell}$-bilinear form $\phi$ such that

$$
\psi(v, w)=\operatorname{Tr}_{E_{\ell / Q_{\ell}}}(\phi(v, w)) \text { for all } v \text { in } V, w \text { in } W .
$$


Proof: $\psi$ defines a $\mathbb{Q}_{\ell}$-linear map $V \otimes_{E_{l}} W \rightarrow \mathbb{Q}_{\ell}$, that is, an element of the $\mathbb{Q}_{\ell}$-linear dual of $V \otimes_{E_{\ell}} W$. But $\operatorname{Tr}_{E_{\ell / Q_{\ell}}}$ identifies the $\mathbb{Q}_{\ell}$-linear dual of $V \otimes_{E_{\ell}} W$ with the $E_{\ell}$-linear dual, and $\psi$ with $\phi$.

Lемма 2.2. Let $\alpha \in E^{*}$ be such that $\bar{\alpha}=-\alpha$. Then there exists a unique $E_{\ell-H e r m i t i a n}$ form $\phi$ on $V_{\ell}$ such that $\psi(v, w)=\operatorname{Tr}_{E_{\ell / Q_{\ell}}}(\alpha \phi(v, w))$ for all $v, w$ in $V_{\ell}$.

Proof: Take $V$ to be $V_{\ell}$ and $W$ to be $V_{\ell}$ with $E_{\ell}$ acting through the involution - Then, by Lemma 2.1, there exists a unique $E_{\ell}$-sesquilinear form $\phi_{1}$ on $V_{\ell}$ such that $\psi(v, w)=\operatorname{Tr}_{E_{\ell / Q_{\ell}}}\left(\phi_{1}(v, w)\right)$.

Let $\phi=\alpha^{-1} \phi_{1}$ be such that $\psi(v, w)=\operatorname{Tr}_{E_{\ell / Q_{\ell}}}(\alpha \phi(v, w))$. Since $\phi$ is sesquilinear, it remains to show that $\phi(v, w)=\overline{\phi(w, v)}$.

By $\psi(v, w)=-\psi(w, v)$ for all $v, \quad w$ in $V_{\ell}, \operatorname{Tr}_{E_{\ell / Q_{\ell}}}(\alpha \phi(v, w))=$ $-\operatorname{Tr}_{E_{\ell / Q_{\ell}}}(\alpha \phi(w, v))=\operatorname{Tr}_{E_{\ell / Q_{\ell}}}(\bar{\alpha} \phi(w, v))$.

Replacing $v$ by $e v$ with $e$ in $E_{\ell}$, one finds that $\operatorname{Tr}_{E_{\ell / \mathbb{Q}_{\ell}}}(\alpha e \phi(v, w))=$ $\operatorname{Tr}_{E_{\ell / \mathbb{Q}_{\ell}}}(\overline{\alpha e} \phi(w, v))$. On the other hand, $\operatorname{Tr}_{E_{\ell / \mathbb{Q}_{\ell}}}(\alpha e \phi(v, w))=\operatorname{Tr}_{E_{\ell / \mathbb{Q}_{\ell}}}(\overline{\alpha e} \overline{\phi(v, w)})$ and as $\overline{\alpha e}$ is an arbitrary element of $E_{\ell}$, the non-degeneracy of the trace implies that $\overline{\phi(v, w)}=\phi(w, v)$. The uniqueness of $\phi$ is obvious from Lemma 2.1.

Lemma 2.3. The commutant of $E_{\ell}$ in $S_{P}\left(V_{\ell}, \psi\right)$ is equal to $U\left(V_{\ell / E_{\ell}}, \phi\right)$.

Proof: Let $T \in \mathrm{Sp}\left(V_{\ell}, \psi\right)$ be such that $T \alpha=\alpha T$ for all $\alpha$ in $E_{\ell}$. Then $T$ can be thought of as an element in $A_{u t} E_{l}\left(V_{l}\right)$. It is easy to check that the map $(v, w) \mapsto \phi(T v, T w)$ is an $E_{\ell}$-Hermitian form.

On the other hand, $\psi(T v, T w)=\psi(v, w)$ is equivalent to $\operatorname{Tr}_{E_{\ell / Q_{\ell}}}(\alpha \phi(T v, T w))=$ $\operatorname{Tr}_{E_{\ell / Q \ell}}(\alpha \phi(v, w))$. By the uniqueness of $\phi$, this amounts to saying that $\phi(T v, T w)=$ $\phi(v, w)$.

REMARK. For those $\ell$ which remain prime in $E, U\left(V_{\ell / E_{\ell}}, \phi\right)$ is an algebraic group over the field $E_{\ell}$. By Weil's restriction of scalars, it can be thought of as a connected algebraic group over $\mathbb{Q}_{\ell}$. For those $\ell$ such that $E_{\ell}=\mathbb{Q}_{\ell} \oplus \mathbb{Q}_{\ell}$, although $U\left(V_{\ell / E_{\ell}}, \phi\right)$ is an algebraic group over $\mathbb{Q}_{\ell}$, it doesn't seem to be obvious that $U\left(V_{\ell / E_{\ell}}, \phi\right)$ is a connected algebraic group.

\section{Proof of the Main Result}

In this section, let $A$ be an abelian variety defined over a number field $K$ where $\operatorname{dim} A=p$ is an odd prime number and $E=\operatorname{End}^{\circ}(A)$ is an imaginary quadratic field. For simplicity, we shall assume the following conditions (by extending the base field $K)$ :

(i) $\operatorname{End}_{K}(A)=\operatorname{End}_{\bar{K}}(A)$. 
(ii) $E \subseteq K \subseteq \bar{K}$ (identifying $E$ as a subfield of $\bar{K}$ ).

(iii) The algebraic envelope $G_{V_{\ell}}$ is connected.

Fix a non-zero element $\alpha$ in $E$ such that $\bar{\alpha}=-\alpha$. Then as in Section 2.3, let $U\left(V_{\ell / E_{\ell}}, \phi\right)$ be the unitary group with respect to the $E_{\ell}$-Hermitian form $\phi$ associated with the Riemann form $\psi$ on the free $E_{\ell}$-module $V_{\ell}$ of rank $p$.

We now prove the main theorem.

THEOREM 3.1. The reductive Lie algebra $\mathcal{S}_{\ell}$ is equal to the Lie algebra of $U\left(V_{\ell / E_{\ell}}, \phi\right)$.

Proof: By Lemma 2.3, it is clear that $\mathcal{S}_{\ell}$ is contained in $\operatorname{Lie}\left(U\left(V_{\ell / E_{\ell}}, \phi\right)\right)$. On the other hand, it is easily seen that $\operatorname{dim}_{\mathbb{Q}_{\ell}} \operatorname{Lie}\left(U\left(V_{\ell / E_{\ell}}, \phi\right)\right)=p^{2}$. It suffices to show that $\operatorname{dim} S_{V_{l}}$ is at least $p^{2}-1$ and the centre of $G_{V_{\ell}}$ is at least of dimension 2.

Now, we divide the rest of the proof into the following steps:

STEP 1. Decomposition of $V_{\ell}$ by the action of $E_{\ell}$.

Let $\bar{V}_{\ell}=V_{\ell} \otimes_{Q_{\ell}} C_{\ell}, \bar{E}_{\ell}=E_{\ell} \otimes_{\mathbb{Q}_{\ell}} C_{\ell}=E \otimes_{\mathbb{Q}} C_{\ell}$, and let $\{\sigma, \tau\}$ be the two embeddings of $E$ into $C_{\ell}$. Corresponding to $\sigma, \tau$, one has an $\bar{E}_{\ell}\left[G_{V_{\ell}}\right]$-module decomposition $\bar{V}_{\ell}=X \oplus Y$. Namely, $X=\left\{v \in \bar{V}_{\ell} \mid e \cdot v=\sigma(e) v\right.$ for all $e$ in $\left.E\right\}$ and $Y=\left\{v \in \bar{V}_{\ell} \mid e \cdot v=\tau(e) v\right.$ for all $e$ in $\left.E\right\}$. Since $V_{\ell}$ is a free $E_{\ell}$-module, both of $X$ and $Y$ are $p$-dimensional $C_{\ell}$-vector spaces.

Let $H$ be the image of the representation $\rho_{\ell}: G_{V_{\ell / C_{\ell}}} \rightarrow G L_{X}$ given by the action of $G_{V_{\ell / C_{\ell}}}$ on $X$.

LEMMA 3.1.1. $H$ is a reductive connected algebraic subgroup of $G L_{X}$ and $\operatorname{End}_{H}(X)=C_{\ell}$. In particular $X$ is an irreducible $H$-module.

Proof: By the theorems of Faltings ([4], Section 5, Satz 3, 4) $G_{V_{\ell / C_{\ell}}}$ acts on $\bar{V}_{\ell}$ and hence on $X$ semisimply. Moreover, one has $\operatorname{End}_{G_{V_{\ell}}\left(Q_{\ell}\right)}\left(V_{\ell}\right)=E_{\ell}$. By $E_{\ell}=$ $C_{\ell} \times C_{\ell}$, one concludes that $\operatorname{End}_{H}(X)=C_{\ell}$.

STEP 2. The Hodge-Tate decomposition of $V_{l}$.

As in Section 2.2, $\bar{V}_{\ell}$ has a Hodge-Tate decomposition $\bar{V}_{\ell}=V_{C_{\ell}}(0) \oplus V_{C_{\ell}}(1)$ with $\operatorname{dim} V_{C_{\ell}}(0)=\operatorname{dim} V_{C_{\ell}}(1)=p$. Here $V_{C_{\ell}}(0)$ is the cotangent space (over $C_{\ell}$ ) to the dual abelian variety $A_{/ C_{l}}$ at its origin. Let $M=V_{C_{l}}(0)$ and $N=V_{C_{l}}(1)$. From condition (ii) of our assumption, both $M, N$ are $\bar{E}_{\ell}$-modules. Accordingly, $M=M_{\sigma} \oplus M_{\tau}$, where $E$ acts via $\sigma$ on the former space and via $\tau$ on the latter. Similarly, one has $N=N_{\sigma} \oplus N_{\tau}$. Let $\operatorname{dim} M_{\sigma}=n_{\sigma}$ and $\operatorname{dim} M_{\tau}=n_{\tau}$. Then $n_{\sigma}+n_{\tau}=p$, where $p \geqslant 3$.

Fix an isomorphism between $C_{\ell}$ and $\mathbb{C}$. Consider the dual module of the $\bar{E}_{\ell^{-}}$ module $\operatorname{Lie}\left(A_{/} C_{\ell}\right)$ (that is the tangent space of $A_{/ C_{\ell}}$ at its origin). By a result of Shimura ([12], Theorem 5), one concludes that both $n_{\sigma}$ and $n_{\tau}$ are positive. 
Lемма 3.1.2. $X=M_{\sigma} \oplus N_{\sigma}$ and $\operatorname{dim} M_{\sigma}, \operatorname{dim} N_{\sigma}$ are relatively prime.

Proof: $\bar{V}_{\ell}=X \oplus Y=\left(M_{\sigma} \oplus M_{\tau}\right) \oplus\left(N_{\sigma} \oplus N_{\tau}\right)$. One sees easily that $X=$ $(X \cap M) \oplus(X \cap N)=M_{\sigma} \oplus N_{\sigma}$. In particular, $\operatorname{dim} N_{\sigma}=n_{\tau}$. Since $n_{\sigma}+n_{\tau}=p$ (odd prime), so $n_{\sigma}, n_{\tau}$ are relatively prime.

Note that Lemmas 3.1.1, 3.1.2 verify the hypotheses of a theorem of Serre ([9], Theorem 3). So we conclude that $H=G L_{X}$. In particular, $\rho_{\ell}$ maps the commutator subgroup of $G_{V_{l / C_{l}}}$ onto $S L_{X}$. This shows that $\operatorname{dim} S_{V_{l}}$ is at least $p^{2}-1$.

STEP 3. The 2-dimensional $C_{\ell \text {-torus }} T_{E_{\ell / c_{\ell}}} \simeq G_{m / c_{\ell}} \times G_{m / c_{\ell}}$. Let $T_{E_{\ell / c_{\ell}}} \simeq$ $G_{m / C_{\ell}} \times G_{m / C_{\ell}}$ be the 2-dimensional torus $\bar{E}_{\ell}^{*}$ over $C_{\ell}$. Recall that $G_{V_{\ell}}\left(C_{\ell}\right)$ is contained in Aut $_{\bar{E}_{\ell}}\left(\bar{V}_{\ell}\right)=G L_{X} \oplus G L_{Y}$. Let $\theta: G_{V_{\ell}}\left(C_{\ell}\right) \subseteq \operatorname{Aut}_{\bar{E}_{\ell}}\left(\bar{V}_{\ell}\right) \stackrel{\text { det }}{\longrightarrow} T_{E_{\ell / C_{\ell}}}$ be the determinant map. Bogomolov ([1], Corollary 1$)$ asserts that $G_{V_{l / C}}$, contains the homotheties $G_{m / c_{\ell}}$. So, the image of $\theta$ contains the diagonal of $G_{m / C_{\ell}} \times G_{m / C_{\ell}}$. On the other hand, the map $\theta \circ h_{V_{l}}: G_{m / C_{\ell}} \stackrel{h_{V_{l}}}{\mapsto} G_{V_{\ell / C_{l}}} \stackrel{\theta}{\rightarrow} T_{E_{\ell / c_{l}}}$ gives $\left(\theta \circ h_{V_{l}}\right)(c)=\left(c^{n_{r}}, c^{n_{\sigma}}\right)$ for all $c$ in $G_{m / c_{l}}$. Since $n_{\sigma} \neq n_{r}$, the image of $\theta \circ h_{V_{l}}$ is distinct from the diagonal of $G_{m / C_{l}} \times G_{m_{/ C_{l}}}$. It follows that $\theta$ is surjective.

So the 2-dimensional torus $T_{E_{\ell / C_{\ell}}}$ is a quotient of $G_{V_{\ell / C_{\ell}}}$. We conilude that the centre of $G_{V_{l}}$ has dimension at least 2 .

This completes the proof of Theorem 3.1.

Corollary 3.2. For all prime dimensional absolutely simple abelian varieties of type IV over number fields,

$$
\mathcal{G}_{\ell}=\mathcal{M} \otimes_{\mathbb{Q}} \mathbb{Q}_{\ell}
$$

Proof: This follows immediately from Theorem 3.1, Theorem 2 in [7], and the result of Taniyama and Shimura in [11]

Corollary 3.3. The Tate conjecture is true for all prime dimensional absolutely simple abelian varieties of type IV over number fields.

Proof: After Faltings proved his theorems ([4], Section 5, Satz 3, Satz 4), it is well-known that if $\mathcal{G}_{\ell}=\mathcal{M} \otimes_{\mathbb{Q}} \mathbb{Q}_{\ell}$, then the conjectures of Hodge, Tate on algebraic cycles (see $[7,15]$ ) over $A(\mathbb{C}), A$ respectively are equivalent. On the other hand, Hodge's conjecture for all prime dimensional absolutely simple abelian varieties of type IV was proved in [14].

Concluding Remark. Let $A$ be a prime dimensional absolutely simple abelian variety over a number field $K$. According to Theorem 2 of Section 21 in [6], one has the following possibilities:

Type I. $\operatorname{dim} A$ is a prime number and $\operatorname{End}_{\bar{K}}(A)=\mathbb{Z}$. 
Type II. $\operatorname{dim} A=2$ and $\operatorname{End}^{\circ}(A)$ is an indefinite quaternion algebra over $\mathbb{Q}$.

Type III. $\operatorname{dim} A=2$ and $\operatorname{End}^{\circ}(A)$ is a definite quaternion algebra over $\mathbb{Q}$.

Type IV. $A$ is as in Section 1.

In his 1984-85 course at Collège de France, J-P. Serre has proved $\mathcal{G}_{\ell} \simeq s p\left(2 d, \mathbb{Q}_{\ell}\right) \oplus$ $\mathbb{Q}_{\ell}$, where $d=\operatorname{dim} A$ is odd and $\operatorname{End}_{\bar{K}}(A)=\mathbb{Z}$. For $\operatorname{dim} A=2$ and $A$ of type II, $\mathcal{G}_{\ell}=\mathcal{M} \otimes_{\mathbb{Q}} \mathbb{Q}_{\ell}$ is well-known (see [2], Corollary 4.2). On the other hand, according to a result of Shimura ([12], Theorem 5), the above Type III abelian variety of dimension 2 doesn't exist. Taking all of these into account, Corollaries 3.2, 3.3 are true for all prime dimensional absolutely simple abelian varieties over number fields.

\section{REFERENCES}

[1] F.A. Bogomolov, 'Sur l'algébricité des représentations l-adiques', C.R. Acad. Sc. Paris, 290 (1980), 701-703.

[2] W. Chi, 'On the $\ell$-adic representations attached to some absolutely simple abelian varieties of type II', J. Fac. Sci. Univ. Tokyo. Sect. IA Math. (1990) (to appear).

[3] P. Deligne, Hodge cycles on Abelian varieties (notes by J.S. Milne): Lecture Notes in Math. 900, pp. 9-100 (Springer-Verlag, Berlin, Heidelberg, New York, 1982).

[4] G. Faltings, 'Endlichkeitssätze fur abelsche varietäten uber zahlkörpern', Invent. Math. 73 (1983), 349-366.

[5] D. Mumford, 'Families of abelian varieties', in Proc. of Symposia in Pure Math. IX, pp. 347-351 (A.M.S., 1966).

[6] D. Mumford, Abelian varieties (Oxford University Press, 1974).

[7] K. Ribet, 'Hodge classes on certain types of abelian varieties', Amer. J. Math. 105 (1983), 523-538.

[8] S. Sen, 'Lie algebras of Galois groups arising from Hodge-Tate modules', Ann. of Math. 97 (1973), 160-170.

[9] J-P. Serre, 'Sur les groupes de Galois attachés aux groupes p-divisibles', in Proceedings of a Conference on Local Fields, pp. 118-131 (Springer-Verlag, Berlin, Heidelberg, New York, 1967).

[10] J-P. Serre, 'Groupes algébriques associés aux modules de Hodge-Tate', Astérisque 65 (1979), 155-188.

[11] G. Shimura and Y. Taniyama, 'Complex multiplication of abelian varieties and its applications to number theory', Publ. Math. Soc. Japan 6 (1961).

[12] G. Shimura, 'On analytic families of polarized abelian varieties and automorphic functions', Ann. of Math. 78 (1963), 149-192.

[13] S.G. Tanke'ev, 'Algebraic cycles on simple 5-dimensional abelian varieties', Math. USSR Izv. 19 (1982), 95-123.

[14] S.G. Tanke'ev, 'Cycles on simple abelian varieties of prime dimension', Math. USSR Izv. 20 (1983), 157-171. 
[15] J. Tate, Algebraic cycles and poles of zeta functions, Arithmetical algebraic geometry, pp. 93-110 (Harper and Row, New York, 1965).

[16] J. Tate, 'p-divisible groups', in Proc. of a Conference on Local Fields, pp. 158-183 (Springer-Verlag, Berlin, Heidelberg, New York, 1967).

Department of Mathematics

National Tsing Hua University

Hsinchu, Taiwan 30043

Republic of China 